\title{
Estudio para la optimización de la composición de un HACFRA (hormigón autocompactante reforzado con fibras de acero) estructural
}

\section{Optimization study of the structural HACFRA composition (Self Compacting Concrete Steel Fiber Reinforced)}

A. Orbe ${ }^{(*)}$, E. Roji ${ }^{(*)}$, J. Cuadrado $^{(*)}$, R. Losada ${ }^{(*)}$

\section{RESUMEN}

El interés de un HACFRA (Hormigón autocompactante reforzado con fibras de acero), radica en la combinación del incremento de capacidad resistente con respecto al hormigón y disminución de la fisuración, aportada por la introducción de fibras de acero, con las ventajas de la puesta en obra que supone la autocompactación.

El artículo analiza la influencia de los diferentes componentes que integran el HACRFA, proponiendo una selección de los mismos, referidos tanto al esqueleto granular como a los diferentes tipos y densidades de fibras de acero, en base a la obtención de sus características y de un comportamiento estructural optimizado.

Palabras clave: Hormigón autocompactante; fibra de acero; comportamiento estructural.

\section{ABSTRACT}

The interest of HACFRA (self compacting concrete reinforced with steel fibers), is the combination of the residual strength increase and cracking decrease compared to plain concrete by the introduction of steel fibers in the mass with the advantages of the self-compacting.

The paper presents an analysis of the influence of different components of the HACRFA and provides their selection, refered to the granular skeleton and to different steel fiber types and amount, in order to obtain an optimization of its features and structural behavior.

Keywords: Self-compacting concrete; steel fiber; structural behavior.

(*) UPV/EHU Universidad del País Vasco - E.T.S. Ingeniería Bilbao (España). Persona de contacto/Corresponding author: eduardo.roji@ehu.es (E. Rojí)

Cómo citar este artículo/Citation: Orbe, A., Rojí, E., Cuadrado, J., Losada, R. (2015). Estudio para la optimización de la composición de un HACFRA (hormigón autocompactante reforzado con fibras de acero) estructural. Informes de la Construcción, 67(537): eo61, doi: http://dx.doi.org/10.3989/ic.13.080.

Licencia / License: Salvo indicación contraria, todos los contenidos de la edición electrónica de Informes de la Construcción se distribuyen bajo una licencia de uso y distribución Creative Commons Reconocimiento no Comercial 3.o. España (cc-by-nc). 


\section{LA CARACTERIZACIÓN DE LOS MATERIALES A EMPLEAR}

\subsection{El hormigón reforzado con fibras}

Los hormigones reforzados con fibras (HRF) no son un concepto de material nuevo, ya que las fibras se han usado como refuerzo desde la antigüedad, desde los pelos de caballo en el mortero hasta los adobes de barro armados con paja.

El hormigón reforzado con fibras (HRF), es un material compuesto por fibras (metálicas, plásticas, fibras de vidrio, etc.) embebidas y distribuidas en la matriz de hormigón. Hay una serie de factores como la geometría de las fibras, su distribución y su densidad en la masa de hormigón, que caracterizan las propiedades y el comportamiento final de la misma.

Ya en el año 1911 Graham añadió fibras al hormigón armado para incrementar su resistencia. En la década de 1960, con la aparición de las fibras sintéticas de nylon, polipropileno y vidrio, surge una nueva etapa del hormigón reforzado con fibras. En la década de los 70 se comenzaron a utilizar en España hormigones reforzados con fibras en aplicaciones diversas tales como la pavimentación de tableros de puentes, pavimentos industriales, contenedores de puertos, revestimientos de túneles, elementos prefabricados, etc. (1). De entre estas aplicaciones, la de revestimientos de túneles o taludes con hormigón reforzado con fibras de acero ha tenido gran éxito, debido sobre todo a sus principales características como la capacidad de absorción de tensiones de tracción y el aumento de la ductilidad (capacidad de absorción de energía).

El uso extendido del HRF en elementos estructurales está encaminado a colaborar en aspectos como:

- El control de la fisuración.

- El comportamiento ante ciclos de carga y descarga.

- La resistencia a impactos.

- Las tracciones debidas a gradientes térmicos.

Las prescripciones y requisitos incluidos en el articulado de la Instrucción de Hormigón Estructural (2) se refieren a hormigones que no incorporan fibras en su masa. Sin embargo, en el Anejo 14 de la misma se establecen unas recomendaciones específicas y complementarias cuando, para mejorar algunas prestaciones, ya sea en primeras edades o en estado endurecido, se empleen fibras en el hormigón, las cuales pueden modificar algunas de sus propiedades.

En ese Anejo las fibras se clasifican, de acuerdo con su naturaleza, en fibras de acero, fibras poliméricas y otras fibras inorgánicas. Los hormigones reforzados con fibras (HRF), se definen como aquellos hormigones que incluyen en su composición fibras cortas, discretas y aleatoriamente distribuidas en su masa.

Las fibras de acero «son elementos rectos y deformados de alambres de acero estirado en frío, recortes de fibras de acero rectas o deformadas, fibras extraídas fundidas, fibras estiradas en frío y fibras molidas de bloques de acero que son adecuadas para su mezcla homogénea con hormigón o mortero» (3).

Al añadir fibras al hormigón, éstas, cortas y discretas, se distribuyen de forma aleatoria (en teoría, ya que en realidad existen ciertos condicionantes que fuerzan algunas disposi- ciones) en la masa, confiriendo a dicha matriz un armado en tres dimensiones que «cose» las fisuras que se producen en el hormigón, transfiriendo los esfuerzos una vez la matriz empieza a fisurarse (4) (Figura 1).

A pesar de que las fibras son elementos discretos, aportan una continuidad, de forma que cuando se fisura no se separa (Figura 2). La incorporación de fibras se hace para mejorar ciertas propiedades específicas del hormigón, tanto en estado fresco, primeras edades o endurecido, y pueden estar presentes con armaduras pasivas y activas. Es de subrayar la mejoría que se produce en el control de la fisuración del hormigón y el aumento de la tenacidad del mismo, lo que lo hace adecuado para soportar acciones dinámicas e impactos.

De una manera general se pueden clasificar las fibras como estructurales y no estructurales.

- Fibras estructurales: aquellas que proporcionan una mayor energía de rotura al hormigón en masa (en el caso de las fibras estructurales, la contribución de las mismas puede ser considerada en el cálculo de la respuesta de la sección de hormigón).

- Fibras no estructurales: aquellas que sin considerar en el cálculo esta energía, suponen una mejora ante determinadas propiedades como por ejemplo el control de la fisuración por retracción, incremento de la resistencia al fuego, abrasión, impacto y otros.

Los parámetros más importantes para la definición de las fibras son la forma de la fibra, su longitud $\left(l_{f}\right)$ y su diámetro equivalente $\left(\mathrm{d}_{\mathrm{f}}\right)$. El cociente entre estos dos últimos constituye un parámetro conocido como esbeltez o relación de aspecto. Los valores de esbeltez habituales oscilan entre 30 y 150 , aunque no es aconsejable pasar de un valor de 100, debido a los problemas que ello conlleva (formación de erizos, atascos en dispositivos de bombeo, etc.). De forma generalizada se ha aceptado que la separación de las fibras no es un factor determinante en el comportamiento del hormigón.

Además, es recomendable que las fibras tengan un módulo de elasticidad al menos tres veces superior al del hormigón (las fibras de acero tienen un módulo de elasticidad 7 veces superior al del hormigón), elevada resistencia a tracción y resistencia a la adherencia, con la matriz del mismo orden o incluso superior a la resistencia a tracción de dicha matriz, y un coeficiente de Poisson y coeficiente de dilatación térmica preferiblemente semejante al de la matriz.

\subsection{El hormigón autocompactante}

Dentro de la caracterización de los hormigones, se puede definir el hormigón autocompactante como «un hormigón que pudiera ser colocado sin utilizar ningún tipo de medios de

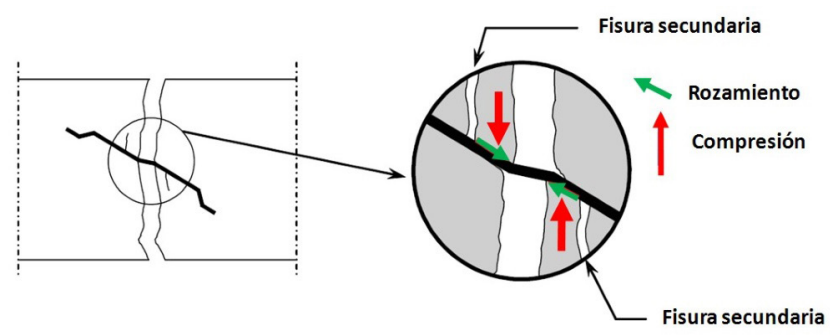

Figura 1. Proceso de transferencia de tensiones en fisura (4). 


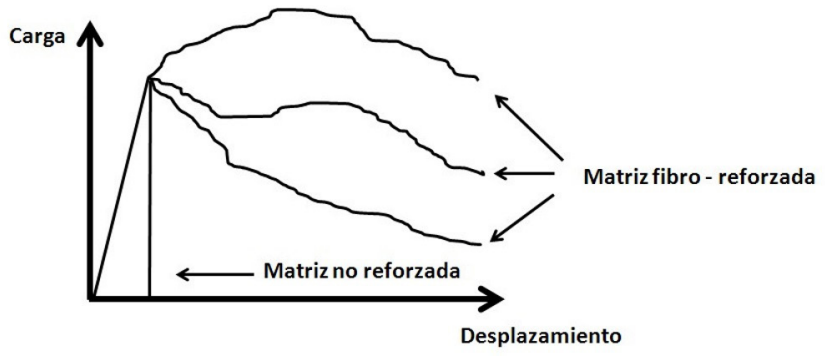

Figura 2. Curvas carga-abertura de fisura.

compactación, y que simplemente por gravedad llenase los moldes de las piezas sin sufrir defectos de hormigonado ni segregación» (5), caracterizado por:

- Capacidad total para el llenado de los moldes o encofrados bajo la acción de su peso propio.

- Ausencia de segregación y de formación de acumulaciones de árido grueso en el atravesado de obstáculos.

- Mantenimiento de homogeneidad y no segregación en las etapas del transporte y la colocación.

Y siendo las principales ventajas de su empleo:

- Eliminación del empleo de elementos de compactación, con el consiguiente ahorro de tiempo y económico que esto implica.

- Excelente calidad del acabado superficial, difícilmente obtenible con hormigones convencionales.

La caracterización de los hormigones autocompactantes en fresco se mide mediante una serie de ensayos específicos, algunos de ellos normalizados (Tabla 1), que referencian sus propiedades plásticas.

\section{ESTABLECIMIENTO DE CRITERIOS PARA LA SELECCIÓN DE COMPONENTES DEL HACFRA}

Los criterios establecidos son el resultado de la investigación llevada a cabo para su aplicación a depósitos cilíndricos de hormigón, de modo que se obtenga la combinación óptima
Tabla 1. Ensayos con hormigones autocompactantes.

\begin{tabular}{|l|l|}
\hline Método de ensayo & \multicolumn{1}{c|}{ Característica Medida } \\
\hline Caja en L & $\begin{array}{l}\text { Capacidad de paso y resistencia frente } \\
\text { a obstrucciones }\end{array}$ \\
\hline Caja en U & $\begin{array}{l}\text { Capacidad de paso y resistencia frente } \\
\text { a obstrucciones }\end{array}$ \\
\hline Anillo en J & $\begin{array}{l}\text { Capacidad de paso y resistencia frente } \\
\text { a obstrucciones }\end{array}$ \\
\hline $\begin{array}{l}\text { Ensayos de } \\
\text { escurrimiento }\end{array}$ & $\begin{array}{l}\text { Resistencia a segregación, estabilidad } \\
\text { y asentamiento } \\
\text { Resistencia a migración de aire } \\
\text { Viscosidad }\end{array}$ \\
\hline $\begin{array}{l}\text { Ensayo de estabilidad } \\
\text { de pantalla }\end{array}$ & $\begin{array}{l}\text { Resistencia al asentamiento y segregación } \\
\text { estática }\end{array}$ \\
\hline Ensayo de bleeding & Resistencia a segregaciones dinámicas \\
\hline Embudo en V & $\begin{array}{l}\text { Capacidad de paso y resistencia } \\
\text { a bloqueos }\end{array}$ \\
\hline
\end{tabular}

entre un hormigón autocompactante y el empleo de fibras estructurales. El interés del hecho de la fabricación de este HACRFA radica en potenciar, gracias al flujo del hormigón generado en el llenado de las estructuras, la orientación de las fibras en la dirección óptima desde el punto de vista de su aportación resistente. Las fibras empleadas en el presente estudio son de acero, considerándose las más apropiadas para la responsabilidad estructural planteada. Los siguientes apartados presentan la metodología empleada para la elección del tipo de fibra entre varias tipologías de ellas y la formulación del hormigón, integrando las fibras y las propiedades autocompactantes requeridas.

\subsection{Elección del tipo de fibra}

Existen en el mercado gran variedad de fibras que pueden considerarse estructurales (Figura 3). Cada tipo de fibra estructural, puede requerir una dosificación diferente, que forme hormigones con propiedades reológicas y resistentes muy diversas.

Del estudio de los conocimientos aportados por determinadas experiencias y pruebas realizadas por diversos investi-

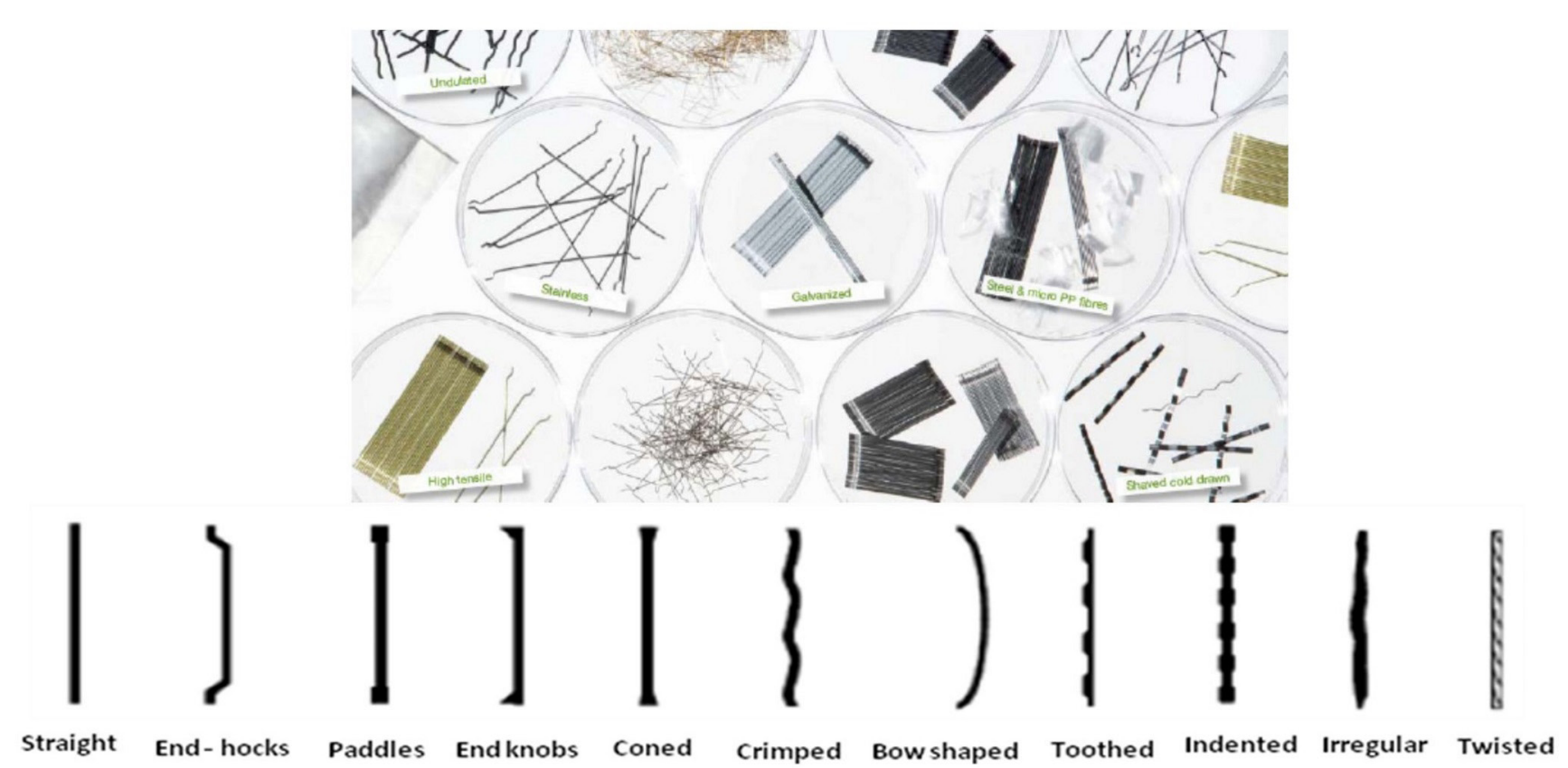

Figura 3. Diferentes tipos y formas de fibras de acero. (Fuente: Bekaert). 


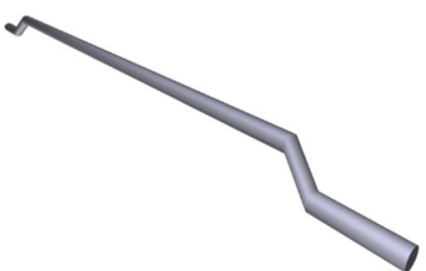

HE

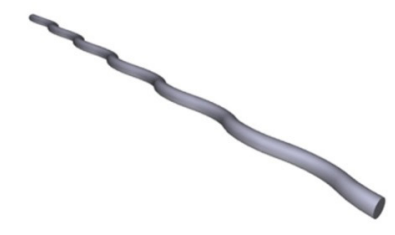

Tabix
Figura 4. Tipologías de las fibras ensayadas.

gadores (6), (7), (8), se preseleccionan únicamente dos tipos de fibras en dos longitudes diferentes, escogiendo fibras de extremos conformados y fibras onduladas con longitudes de 50 y $60 \mathrm{~mm}$ en ambos casos (Figura 4).

Las fibras empleadas son del fabricante ArcelorMittal, cuyas designaciones y propiedades se resumen en la siguiente tabla (Tabla 2).

En las fibras tipo HE, las dimensiones del extremo conformado no varían entre las dos longitudes ensayadas. Se presupone que una longitud mayor únicamente presentará una mejora debido al mecanismo de adherencia. En el caso de las Tabix, la longitud de onda tampoco se modifica. Para este tipo de fibra, a mayor longitud de la fibra aumenta el esfuerzo a tracción soportado, dado que se incrementa la longitud de adhesión, pero disponiéndose también de más ondulaciones para su anclaje.

La selección del modelo de fibra se basa en los resultados obtenidos en un conjunto de ensayos a arrancamiento de las mismas, sobre una serie de modelos diseñados a tal efecto (Figura 5) (9). Se debe hacer constar, sin embargo, que este ensayo permite determinar cuál es la fibra más acorde a las condiciones de uso previstas, pero no es representativo del HACRFA; es decir, los resultados obtenidos no son adecuados para el diseño del material compuesto, que debe ser analizado en conjunto.

Dado que la fibra deslizará por el extremo menos embebido, éste no será superior a la mitad de la longitud de la fibra, adoptando para el ensayo un valor medio igual a la cuarta parte de su longitud.

El comportamiento de cada tipo de fibra presenta una alta dispersión. La curva carga-desplazamiento puede ser fácilmente alterada debido a ligeros cambios en la inclinación y la longitud embebida de la fibra.
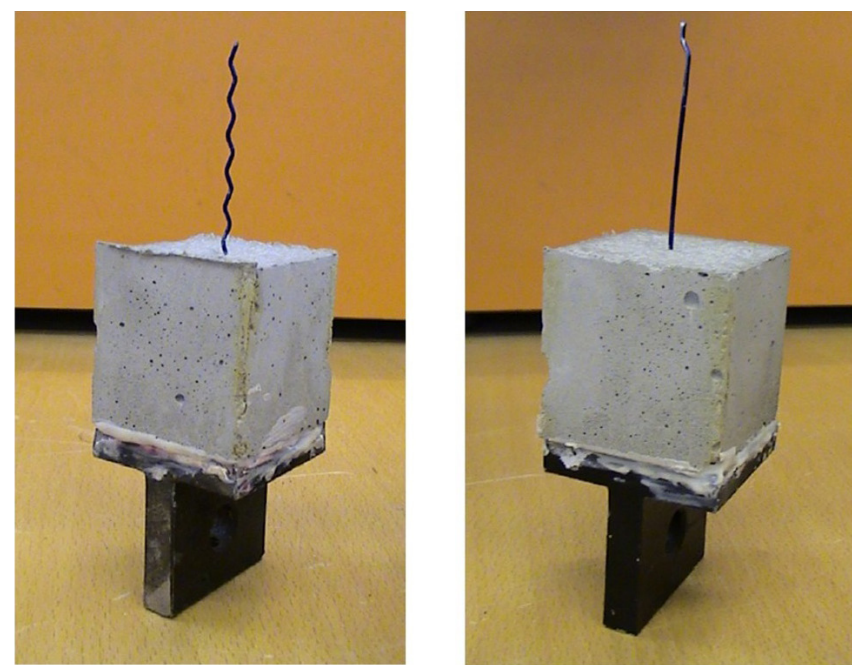

Figura 5. Modelos para ensayo de arrancamiento.

Investigaciones previas constatan que las fibras onduladas presentan mayores capacidades de transmisión de carga en cuanto a longitudes elevadas se refiere, respecto a los resultados aportados por las fibras de extremos conformados (10).

Mientras que las fibras onduladas requieren de mayores longitudes para desarrollar toda su capacidad, es decir, mayor número de ondas embebidas, las fibras con los extremos conformados básicamente necesitan tener embebido el gancho para su correcto anclaje y que mayores longitudes no aportan un incremento sustancial de la capacidad portante, tal y como ya se menciona en (7).

En los ensayos realizados se decidió el empleo de acero de bajo contenido en carbono, dado que las cargas bajo las que se produce el deslizamiento de la fibra son notablemente menores a las que producirían su rotura.

Por lo tanto, y en concordancia con todo lo anteriormente expuesto, se optó por emplear las fibras de extremos conformados de longitud más reducida, cuya denominación se corresponde con $\mathrm{HE} 1 / 50$, de acuerdo a su fabricante.

Los resultados de los ensayos, para los tipos de fibra seleccionadas, se representan en las gráficas carga-desplazamiento siguientes (Figura 6) (9).

Las fibras utilizadas en la investigación son las más comúnmente empleadas en el ámbito de la construcción, con simila-

Tabla 2. Características de las fibras empleadas.

\begin{tabular}{|l|c|c|c|c|}
\hline \multicolumn{1}{|c|}{ Designación } & HE 1/50 & HE+ 1/6o & Tabix 1/50 & Tabix+ 1/6o \\
\hline Geometría & Extremos & Conformados & \multicolumn{2}{c|}{ Ondulada } \\
\hline Longitud (mm) & 50 & 60 & 50 & 60 \\
\hline Diámetro (mm) & 1,00 & 1,00 & 1,00 & 1,00 \\
\hline Longitud de onda (mm) & N/A & N/A & 8 & 8 \\
\hline Amplitud de onda (mm) & N/A & N/A & $0,40-0,65$ & $0,40-0,65$ \\
\hline Longitud de gancho (mm) & $1-4$ & $1-4$ & N/A & N/A \\
\hline Profundidad de gancho (mm) & 1,80 & 1,80 & N/A & N/A \\
\hline Relación de aspecto & 50 & 60 & 50 & 60 \\
\hline Resistencia a tracción $(\mathrm{MPa})$ & 1100 & 1450 & 1100 & 1450 \\
\hline Número de fibras por kg & 3100 & 2600 & 3100 & 2500 \\
\hline Longitud embebida (mm) & 1,25 & 1,50 & 1,25 & 1,50 \\
\hline
\end{tabular}




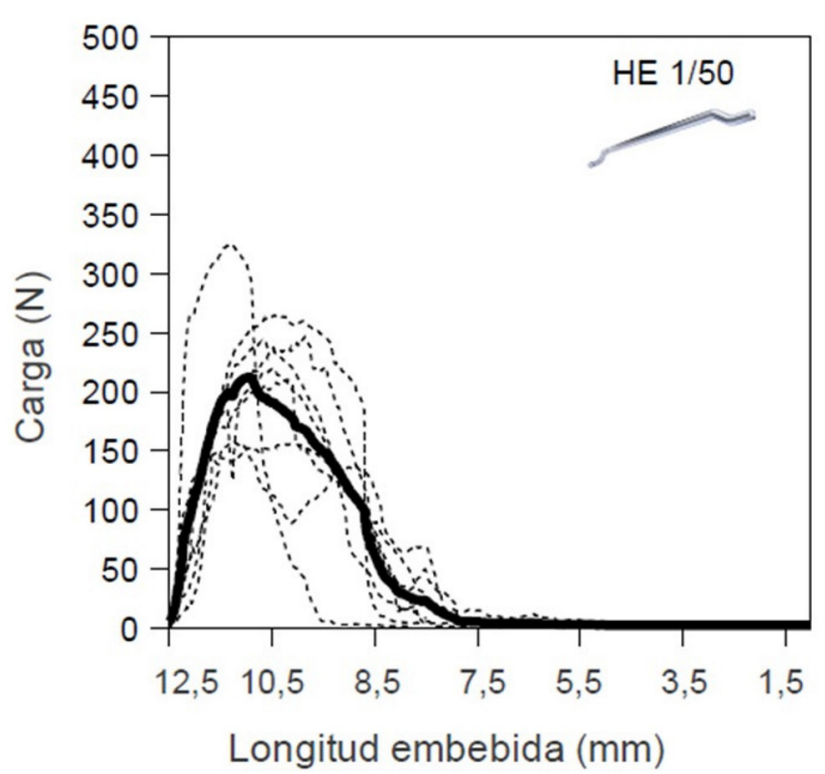

(a) $\operatorname{HE} 1 / 50$

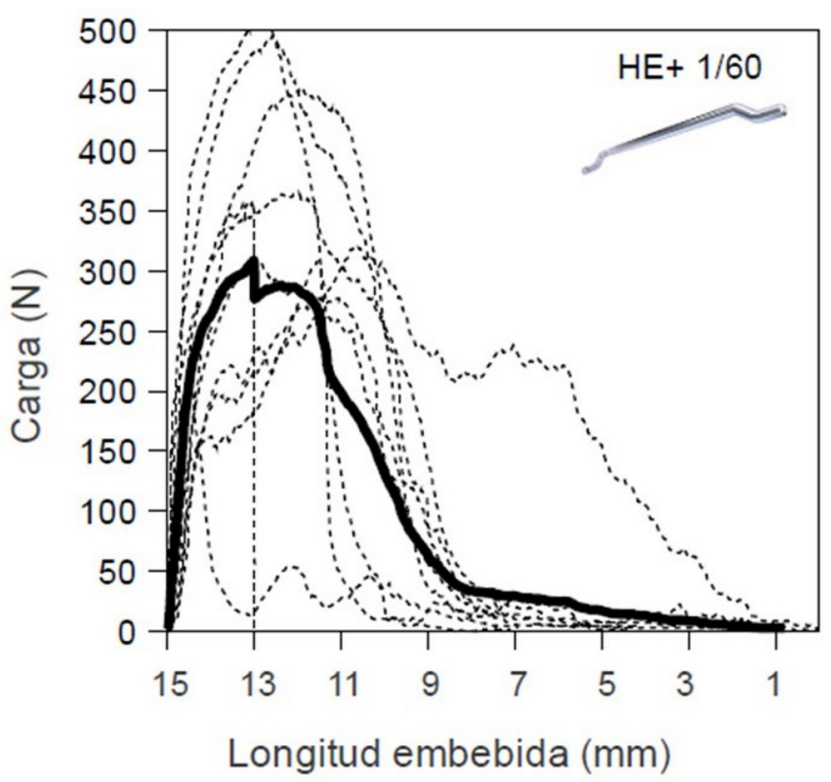

(b) $\mathrm{HE}+1 / 60$

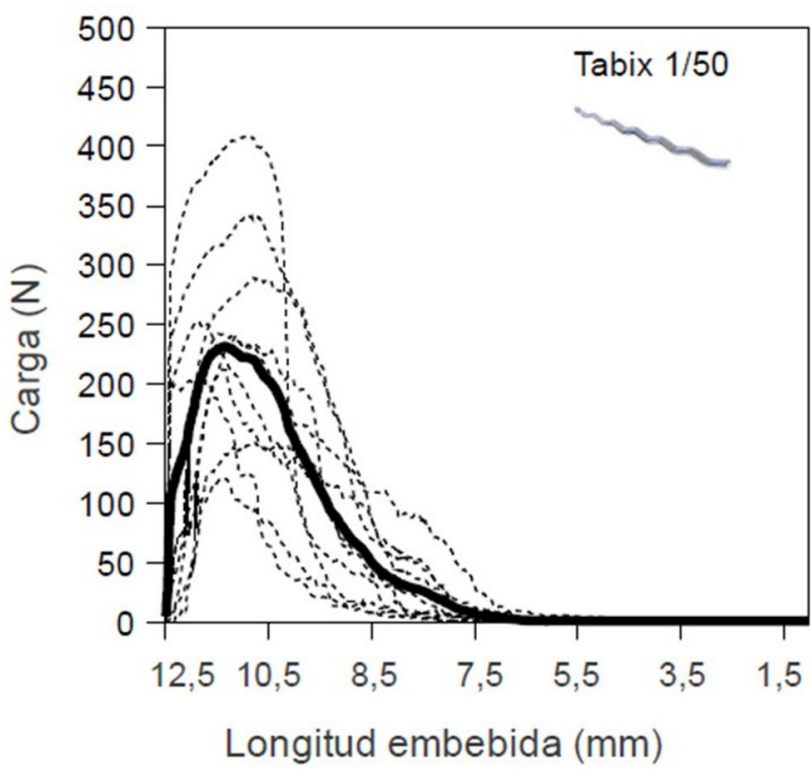

(c) Tabix $1 / 50$

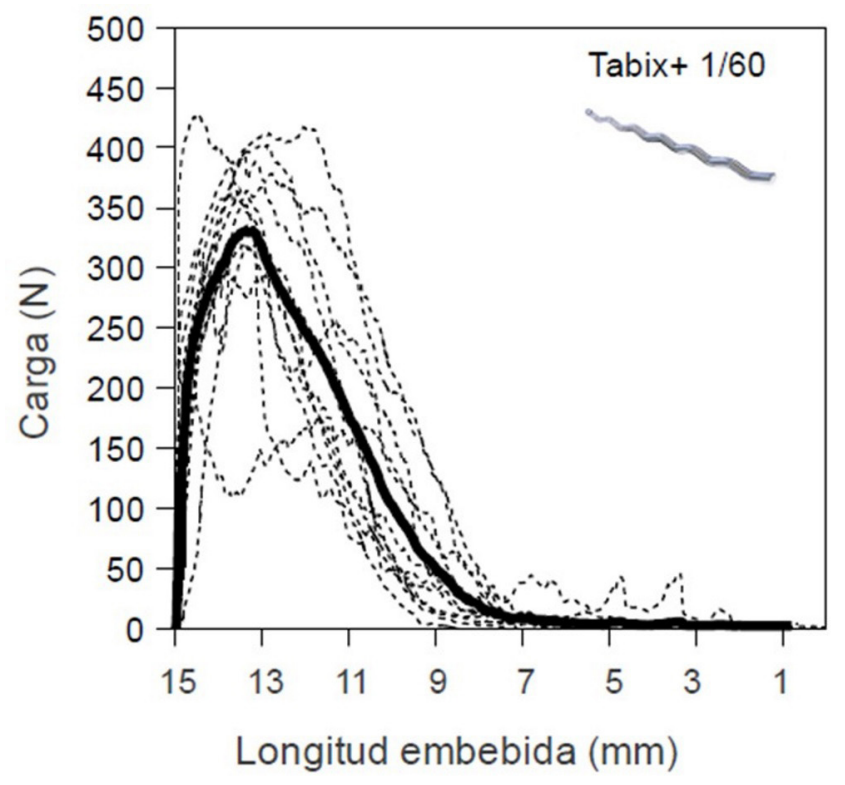

(d) Tabix $+1 / 60$

Figura 6. Relaciones carga-desplazamiento para los tipos de fibras.

res resistencias frente al arrancamiento en cuanto a longitudes embebidas reducidas se refiere.

Se comprueba que las fibras, una vez extraídas de la matriz de hormigón, han sufrido estiramientos claramente apreciables.

Se demuestra que el mecanismo de arrancamiento prevalece frente al de rotura para la aplicación estudiada. Independientemente de la forma, la relación de aspecto es uno de los parámetros más influyentes en la dosificación, así como la posibilidad de formarse erizos de fibras durante el amasado.

El rango de relaciones de aspecto para los tipos de fibras más habituales, oscila entre 80 y 38 , en función de su longitud y diámetro. La fibra empleada se encuentra en la mitad de dicho rango. Por tanto, se considera más adecuado emplear fibras más cortas y numerosas por unidad de volumen, que contribuyan de manera más eficiente al control de fisuración y a resistencia a tracción de manera simultánea.

\subsection{Propuesta de formulación óptima del hormigón}

El hormigón autocompactante reforzado con fibras de acero se ha dosificado en función de las prestaciones necesarias para su aplicación a depósitos circulares de contención, elementos de muros de reducido espesor, donde interesa conseguir la máxima orientación en sentido horizontal de las fibras 
dentro de la masa, puesto que los principales esfuerzos de tracción se originan en dicha dirección.

Si bien el estudio realizado no deja de ser un planteamiento teórico, resulta imprescindible determinar una ubicación hipotética del proyecto, dado que las plantas de suministro de hormigón, en cada localización geográfica, emplean materias primas de características, en algunos casos, realmente dispares.

Con dicho motivo, la formulación del hormigón se ha establecido en base a una estrecha relación con la empresa Financiera y Minera (FyM), del grupo Italcementi. Se ha determinado que la obra se ejecutará en la zona de influencia de su planta de Kukularra situada en Erandio (Bizkaia). De este modo, los áridos a emplear serán los habituales utilizados en la planta y su fabricación se realizará en base a los procedimientos ordinarios establecidos en su sistema de gestión. Se dispone, por tanto de áridos calizos procedentes de machaqueo con un tamaño o/4 para la arena y 4/11 para la gravilla. El cemento se corresponde con un CEM II mixto con adiciones de escoria siderúrgica y filler calizo, una resistencia a compresión característica de 42,5 MPa y altas resistencias iniciales (CEM II/A$\mathrm{M}(\mathrm{S}-\mathrm{L})$ 42.5R). Las fibras se corresponden con las designadas como HE $1 / 50$.

Tal y como señala (11), aun existiendo diversos métodos de dosificación, éstos no son generalizables y las empresas del sector han establecido sus propias metodologías para obtener mejoras desde el punto de vista competitivo.

De acuerdo a la experiencia recogida a lo largo de los años por la empresa suministradora de hormigón, se estima que para los áridos que se manejan en el entorno señalado, cada metro cúbico de hormigón autocompactante debe contener alrededor de 1000 kilogramos de pasta. Esta pasta está compuesta de cemento, agua y la fracción de arena que atraviesa el tamiz de abertura igual a 1 milímetro.

Como consecuencia de la aplicación planteada, según (2), se debe dosificar un hormigón acorde a un ambiente correspondiente a clases específicas de exposición $\mathrm{Q}_{\mathrm{b}}$ o $\mathrm{Q}_{\mathrm{c}}$, en función del grado de agresividad, con un contenido mínimo en cemento de $350 \mathrm{~kg} / \mathrm{m}^{3}$ y una máxima relación agua/cemento

Tabla 3. Granulometrías de los áridos.

\begin{tabular}{|c|c|c|c|}
\hline Tamiz & Arena o/4 & Grava 4/11 & Combinada \\
\hline 31,5 & 100,0 & 100,0 & 100,0 \\
\hline 25 & 100,0 & 100,0 & 100,0 \\
\hline 22,4 & 100,0 & 100,0 & 100,0 \\
\hline 20 & 100,0 & 100,0 & 100,0 \\
\hline 16 & 100,0 & 100,0 & 100,0 \\
\hline 14 & 100,0 & 100,0 & 100,0 \\
\hline 12 & 100,0 & 93,5 & 97,5 \\
\hline 10 & 100,0 & 52,6 & 81,5 \\
\hline 8 & 100,0 & 19,7 & 68,7 \\
\hline 4 & 100,0 & 5,10 & 63,0 \\
\hline 2 & 66,1 & 2,20 & 41,2 \\
\hline 1 & 42,3 & 2,00 & 26,6 \\
\hline 0,5 & 27,9 & 1,50 & 17,6 \\
\hline 0,25 & 19,7 & 1,50 & 12,6 \\
\hline 0,125 & 14,8 & 1,50 & 9,60 \\
\hline 0,063 & 11,4 & 1,70 & 7,60 \\
\hline
\end{tabular}

más restrictiva en el segundo caso, siendo éste un valor de 0,45. Las granulometrías de los áridos (Tabla 3) muestran que la arena aporta un $42,3 \%$ de su peso a la pasta.

Aceptando que la parte de arena oscila entre 1000 y $1500 \mathrm{~kg}$ en función de las prestaciones requeridas para el hormigón final, en la investigación realizada se opta por una dosificación de $1100 \mathrm{~kg}$ de arena, de los cuales unos $465 \mathrm{~kg}$ formarán parte de la pasta.

Para aproximarse a los $1000 \mathrm{~kg}$ de pasta mencionados inicialmente, se requiere iterar con la cantidad de cemento y agua, sin superar los límites establecidos de contenido mínimo de cemento y máxima relación agua/cemento. De esta manera se establecen las cuantías mostradas en la Tabla 4. Con dichos valores se puede estimar un peso de pasta de aproximadamente 1070 kilogramos, con una cantidad de arena dentro de los márgenes marcados.

Otras recomendaciones (12) indican que es necesario un contenido total de finos cercano a los $450-600 \mathrm{~kg} / \mathrm{m}^{3}$, lo cual se cumple también en este caso con alrededor de $590 \mathrm{~kg} / \mathrm{m}^{3}$. La granulometría de la misma cumple los requisitos establecidos en la normativa respecto a los límites superiores e inferiores, por lo que no resulta necesario mezclarlo con arena de corrección, por lo general, de origen silíceo y tamaño o/1.

Debido a que se ha establecido por diseño el empleo de 50 kilos de fibras por cada metro cúbico de hormigón, se considera como un agregado más a tener en cuenta en la búsqueda del esqueleto granular más compacto, a fin de obtener un hormigón de adecuadas prestaciones (resistencia y durabilidad).

Para ello se sigue el procedimiento establecido en la norma ASTM C29/C29M, mezclando los agregados en seco, rellenando un recipiente de peso y volumen conocidos con la mezcla, compactándola y, a continuación, realizando su pesaje. La mayor compacidad corresponderá con la proporción que muestre la mayor densidad, o lo que es equivalente, un menor volumen de huecos (Figura 7).

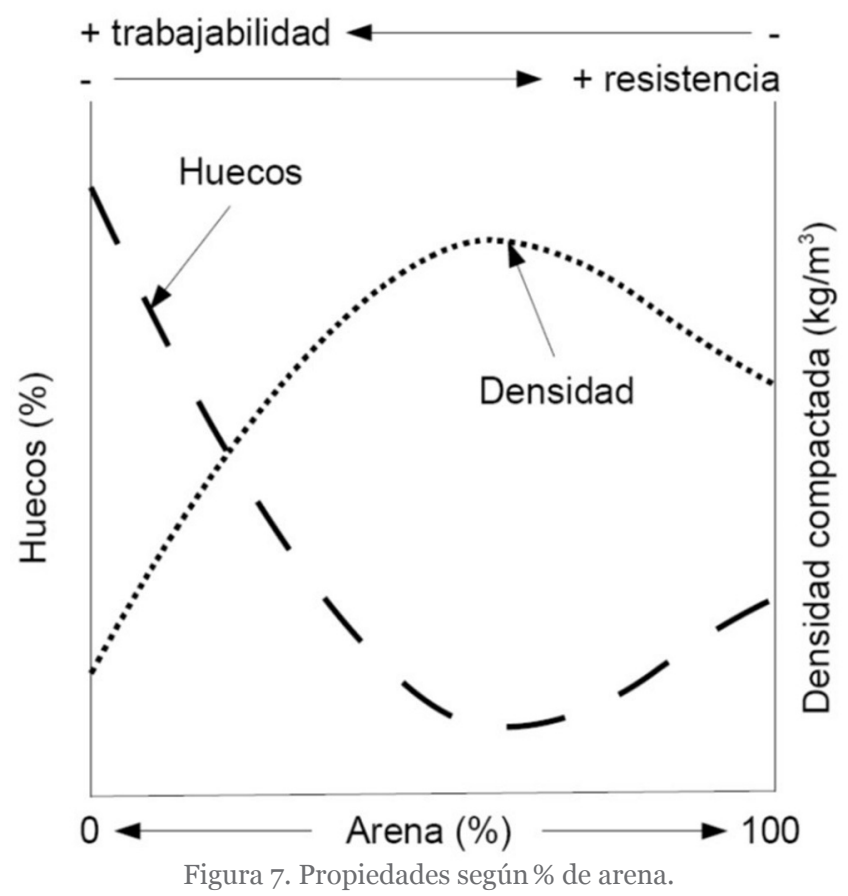



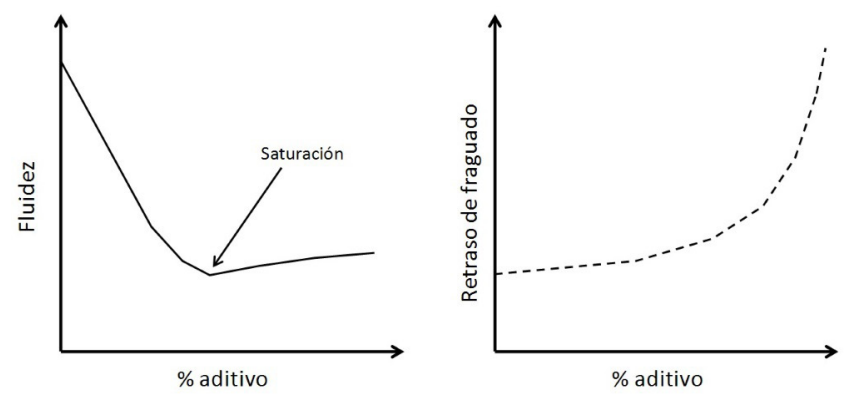

Figura 8. Efectos por sobredosis de aditivo.

\subsection{Consideraciones para la selección de aditivos}

La determinación de los aditivos, se realiza de manera empírica en base a los habituales ensayos de caracterización de los hormigones autocompactantes, empleándose para este caso concreto, el ensayo de escurrimiento y el embudo en V. En esta fase se han añadido a la masa dos aditivos, uno polifuncional y otro superplastificante, que ejerce la labor de reductor de agua de alta actividad. La combinación de ambos permite optimizar la solución final.

El introducir inclusiones de aire, permite mejorar la reología de la masa y contrarrestar posibles segregaciones principalmente de las fibras, que son los agregados más esbeltos. Las proporciones de ambos tipos de aditivo, dependen también de la estación, calurosa o fría, en la que se realiza la amasada, puesto que puede originarse un retraso excesivo en el fraguado, resultando no aceptable.

La proporción de aditivo polifuncional se encuentra cerca de su límite superior, de forma que trabaje como superplastificante-aireante, pero sin provocar intensos retrasos ni oclusiones de aire importantes. Un excesivo aporte de aditivos no aumenta infinitamente la fluidez del hormigón, puesto que existe un punto de saturación a partir del cual se mantiene constante y puede generar un no deseado retardo (Figura 8). Por su parte conviene controlar el porcentaje de superplastificante debido a su coste, ya que una sobredosis puede acarrear la segregación de los agregados. El porcentaje de aire del hormigón, por su parte, se limita a un $2 \%$.

\section{DEFINICIÓN DE UN HACFRA OPTIMIZADO}

Los resultados de los ensayos realizados establecen que las propiedades óptimas se consiguen con el empleo de una combinación de aditivo polifuncional y superplastificante en unas proporciones aproximadas de $0,93 \%$ y $1,12 \%$, respectivamente (Tabla 4). Al mismo tiempo, se garantiza una adecuada resistencia y durabilidad gracias a que permite emplear una reducida cantidad de agua de amasado.

El procedimiento de mezcla empleado comienza con la mezcla en la amasadora de la arena y la grava. Con posterioridad se incorpora el cemento y se continúa amasando en seco. Cuando la distribución resulte homogénea, se adicionan 3/4 partes del agua de amasado y se prosigue con el mezclado. Se aportan los aditivos con la parte restante de agua y se amasa unos minutos para que los aditivos hagan efecto. Finalmente, se añaden las fibras y se persiste en el amasado para la completa y correcta distribución de las mismas en la matriz de hormigón.

La Figura 9 muestra la secuencia de imágenes correspondientes con el inicio del ensayo de escurrimiento, el tiempo en el que se alcanza el diámetro de $500 \mathrm{~mm}\left(\mathrm{~T}_{50}\right)$ y el diámetro final obtenido $\left(\mathrm{D}_{\mathrm{f}}\right)$ para la dosificación teórica diseñada en el laboratorio.

El contenido mínimo en cemento establecido como dato de partida oscila entre 325 y $350 \mathrm{~kg} / \mathrm{m}^{3}$ con una relación agua cemento máxima de 0,45 , en función de una clase específica de exposición a ambiente químico agresivo $\left(\mathrm{Q}_{\mathrm{a}}, \mathrm{Q}_{\mathrm{b}}\right.$ o $\left.\mathrm{Q}_{\mathrm{c}}\right)$ debido a la posibilidad de contener aguas residuales, por ejemplo, en la aplicación del depósito cilíndrico propuesto. Ello da lugar, generalmente, a la obtención de resistencias a compresión cercanas a 30 o $35 \mathrm{MPa}$. Partiendo de la base que los hormigones autocompactantes tienden a presentar, debido a su dosificación y además de lo expresado en relación con el ambiente al que estará expuesta la estructura, resistencias superiores a los límites inferiores permitidos por la normativa, se diseña, por tanto, un hormigón de resistencia moderada de $52 \mathrm{MPa}$.

La reducción en la trabajabilidad de la masa que originan las fibras, queda compensada en cierta medida al utilizarse un hormigón autocompactante y con el empleo adecuado de aditivos superplastificantes. La importancia radica, por tanto en establecer la dosificación óptima del HAC.

$\mathrm{Al}$ igual que los áridos de forma laminar o acicular que presentan bajos coeficientes de formas o elevados índices de lajas, las fibras demasiado esbeltas requieren de mayor cantidad de cemento y pueden originar un amasado incorrecto, afectando negativamente a las propiedades del hormigón endurecido.

\section{A MODO DE CONCLUSIÓN}

El presente artículo muestra una sencilla metodología para dosificar un HACFRA con materiales convencionales. Es necesario remarcar que el conocimiento de los materiales y sus granulometrías resulta imprescindible para un resultado óptimo.

El contenido de humedad es una variable cuya influencia es notoria, sobre todo en lo que respecta a la segregación.

Aunque la cantidad de cemento pueda parecer elevada, su aplicación en ambientes con clase de exposición específica (Qa, Qb y Qc) resulta interesante económicamente, ya que la diferencia se ve notablemente reducida. Profundizar en esta línea de trabajo podría pasar por incluir la adición de finos de subproductos (cenizas volantes, filler calizo, etc.), en detrimento de la cantidad de cemento aportada, con el consiguiente aumento de interés tanto económico como medioambiental.

Tabla 4. Dosificación teórica.

\begin{tabular}{|c|c|c|c|c|c|c|c|}
\hline Componente & $\begin{array}{c}\text { Cemento } \\
\text { II 42.5R }\end{array}$ & $\begin{array}{c}\text { Arena } \\
\mathbf{0} / \mathbf{4}\end{array}$ & $\begin{array}{c}\text { Grava } \\
\mathbf{4 / 1 1}\end{array}$ & $\begin{array}{c}\text { Fibras } \\
\text { HE 1/50 }\end{array}$ & Agua & $\begin{array}{c}\text { Aditivo } \\
\text { Poli. }\end{array}$ & $\begin{array}{c}\text { Aditivo } \\
\text { SP }\end{array}$ \\
\hline $\mathrm{Kg} / \mathrm{m}^{3}$ & 430 & 1100 & 725 & 50 & 175 & 4,00 & 4,80 \\
\hline
\end{tabular}




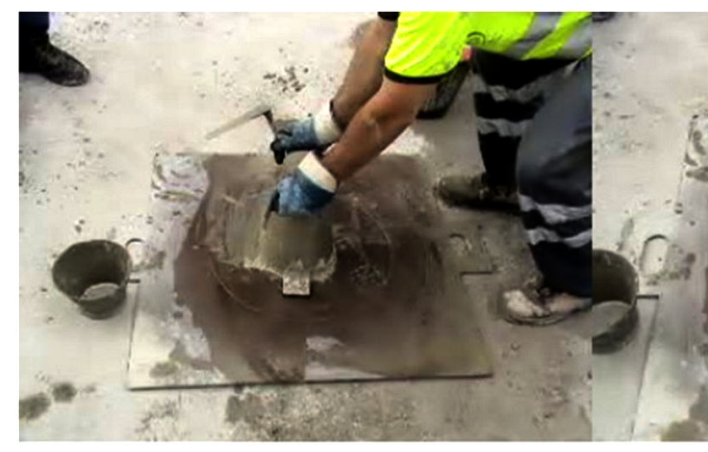

(a) $\mathrm{t}=0 \mathrm{seg}$

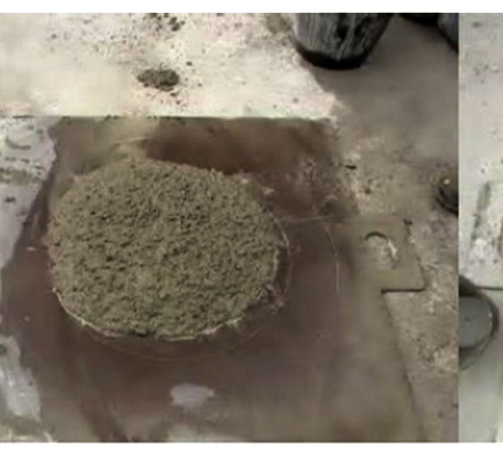

(b) $\mathrm{t}=9 \mathrm{seg}$

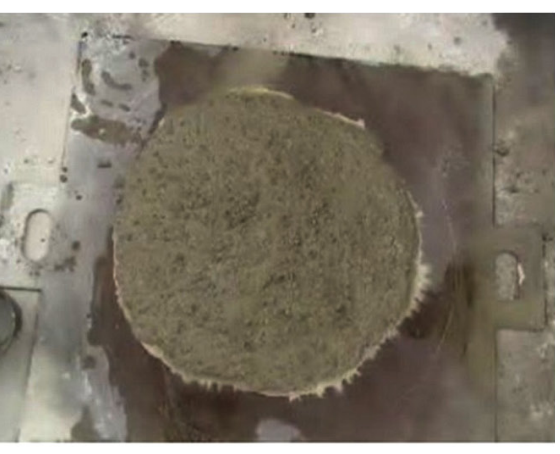

(c) $\quad \mathrm{t}=24 \mathrm{seg}$

Figura 9. Ensayo de escurrimiento de amasada en laboratorio.

De acuerdo con los resultados obtenidos en los ensayos realizados, la resistencia al arrancamiento de las fibras individuales, se debe medir en longitudes embebidas acordes a los mecanismos de deslizamiento que se generarán en situaciones reales. La longitud embebida de la fibra oscilará entre o y L/2, siendo L la longitud de la fibra, con un valor medio de $\mathrm{L} / 4$. Para ciertos tipos de geometrías, un aumento de la longitud embebida no mejora sustancialmente la resistencia frente al arrancamiento, por lo que resultará más interesante optar por las que, con el mismo diámetro son más cortas, al objeto de disponer del mayor número de ellas en las secciones fisuradas. Para las aplicaciones convencionales y resistencias moderadas, resulta suficiente el empleo de fibras con bajo contenido de carbono.
Hoy en día, sigue resultando inevitable comprobar la dosificación mediante ensayos normalizados, de forma que se asegure un adecuado comportamiento reológico del material.

\section{AGRADECIMIENTOS}

- Proyecto de Investigación del MEC: «Cuantificación de la sostenibilidad en ingeniería de la construcción con y sin incertidumbre. Aplicación y contraste en diferentes componentes y escalas constructivas» (BIA2010-20789-Co4-04).

- Grupo de Investigación Consolidado por Gobierno Vasco IT781-13: Sostenibilidad integral en sistemas de edificación y sus materiales.

\section{REFERENCIAS}

(1) Orbe A., Cuadrado J., Losada R., Rojí E., Maturana A. (2011). Desarrollo estructural del Hacfra: hacia una caracterizacion de su composicion. En V Congreso de ACHE. Barcelona

(2) Ministerio de Fomento. (2008). Instrucción de Hormigón Estructural EHE-o8.

(3) AENOR. (2008). UNE-EN 14889-1 Fibras para hormigón. Parte 1: Fibras de acero. Asociación Española de Normalización.

(4) Narayan, N. (2013). Steel Fibre Reinforced Concrete (SFRC): Areas of Application. The Masterbuilder. www.masterbuilder.co.in.

(5) Fernández J., Burón M. (2005). Guía práctica para la utilización del hormigón autocompactante. Madrid: IECA.

(6) Maturana, A. (2013). Estudio teórico-experimental de la aplicabilidad del hormigón reforzado con fibras de acero a losas de forjado multidireccionales (PhD thesis). UPV/EHU.

(7) Cunha,V., Barros, J., Sena-Cruz, J. (2010). Pullout behavior of steel fibers in self-compacting concrete. Journal of Materials in Civil Engineering, 22(1):19, doi: http://dx.doi.org/10.1061/(ASCE)MT.1943-5533.0000001.

(8) Robins, P., Austin, S., Jones, P. (2002). Pull-out behaviour of hooked steel fibres. Materials and Structures, 35: 434442.

(9) Orbe, A., Cuadrado, J., Losada, R., Rojí, E. (2012). Framework for the design and analysis of steel fiber reinforced selfcompacting concrete structures. Construction and Building Materials, 35: 676-686, doi: http://dx.doi.org/10.1016/j. conbuildmat.2012.04.135.

(10) May, M. (2008). Änderung des arbeitsverhaltens von stahlfaserbetonen mit hochfesten stahlfasern. Projektarbeiten. Dresden: Hochschule für Technik und Wirtschaft.

(11) García, J. (2004). Diseño de hormigones dirigido a la aplicación (Master's thesis). Universitat Politècnica de Catalunya.

(12) ACHE. (2008). Hormigón Autocompactante: Diseño y Aplicación. Technical Report. Asociación Científico-Técnica del Hormigón Estructural. 